\title{
Billiards Inspired Optimization Algorithm for Optimal Antenna Selection
}

\author{
Aditya Killekar \\ University of Southern California, USA \\ killekar@usc.edu
}

\begin{abstract}
In both the telecommunication industry and academia, massive Multiple-Input Multiple-Output (MIMO) is a highly developed technology in wideband wireless communication system's prospects that incite widespread concentration. In the massive MIMO system, pilot contamination is considered a basic problem. The modeling of wireless systems possesses an important problem over system throughput. Even though, environmental security, and energy-saving, considered predictable developments and universal demands. Therefore, by representing all these results, this work aims to develop a novel billiards-inspired optimization technique to choose the best transmit antennas chosen by taking into consideration of multi-objective issue which increases both relative energy effectiveness and capability. Actually, the developed method optimally tunes a number of transmit antennas and decides which antenna to be chosen. At last, proposed work performance is evaluated and shown with existing techniques with respect to the relative efficiency analysis, capacity analysis, and optimal antenna selection analysis.
\end{abstract}

Keywords: MIMO, TAS, Energy Efficiency, CSI, Optimization Algorithm

\begin{tabular}{ll} 
Nomenclature & \\
\hline Abbreviation & Description \\
\hline MIMO & Multiple-Input Multiple-Output \\
TAS & Transmit Antenna Selection \\
EE & Energy Efficiency \\
SIC & Successive Interference Cancellation \\
SNR & Signal to Noise Ratio \\
RF & Radio Frequency \\
CSI & Channel State Information \\
BS & Base Station \\
SISO & Single-Input Single-Output \\
\hline
\end{tabular}

\section{Introduction}

Nowadays the requirement of high transmission data rates has to turn out to be a serious problem with the overview of wireless communication systems. The exploitation of MIMO and multimode antennas has appeared as a motivating scheme to raise throughput and capability of wireless systems in prosperous scattering environments [1].

Multiple antennas or MIMO systems have been proposed to improve the communication systems performance by either skirmishing or using the multi-path scattering in the wireless channel, and extensively used within wireless standards, for instance, IEEE LTE and 802.11ac. Even though MIMO approaches enhance the facility of wireless systems in similar transmit power budget, and requisite bandwidth, circuit energy utilization of MIMO systems, is therefore superior to that of SISO systems. MIMO systems with existing supportive relay communications obtained important research attention because of maximum data rates, enhanced spectral effectiveness, improved coverage area, enhanced capability and maximum diversity gain [2].

At the receiver, CSI and the transmitter are needed to attain high performance in MIMO systems. Therefore, more frequency or time resources are exhausted on signaling overhead. Additionally, it is hard to attain CSI precisely and entirely. Hence, the performance of EE is enhanced in partial CSI, or impairments of CSI turn out to be a significant problem. The imperfect CSI effect was modeled as 
interference and iterative techniques were presented to resolve optimum EE. Estimation of capacity in faulty CSI at the transmitter and ideal CSI at the receiver was exploited to compute EE. Moreover, robust multiuser beamforming was modeled to increase EE for multicell networks in attendance of CSI imperfection at the Base Station [4].

Current studies are concentrated towards the practice of under-used ultra-high frequency spectrum generally called $\mathrm{mmW}$ band. Nevertheless, with high path loss communication is coupled at such high frequencies. Beamforming, and Precoding methods exploiting large antenna arrays, can offer adequate array increase to surmount free space path loss and found communications with satisfactory SNR. As the count of transmit antennas is enormous in massive MIMO model operating at $\mathrm{mmW}$ frequencies, completely digital precoding which permits the utmost advantages of spatial multiplexing needs a detached RF chain for each antenna that is not almost possible because of high power and energy utilization with maximum complexity [15].

The primary objective of this article is to develop a novel billiards-inspired optimization approach to choose optimal transmit antenna selection.

- This is performed by taking into consideration of a multi-objective problem that raises both the relative EE and capacity.

- It seems that the developed method optimally adds up the transmit antennas and promotes recognizes suitable antenna to be chosen.

- Finally, developed method performance is examined and validated with conventional techniques in the perspective of relative efficiency analysis, capacity analysis, and analysis of optimal antenna selection.

\section{Related Works}

In 2020, Mahdi Eskandar et al [1], considered the maximization of allocation of power and EE problem in point-to-point, spatial multiplexing of MIMO schemes. Conversely, existing EE optimization techniques that determine the loading of power in ideal CSI assumption, energy-efficient equivalent power allocation was taking into consideration. It desires at transmitter limited feedback and also for optimal EE derived a lower bound expression regarding the parameters of the system. In 2020, Mostafa parvin et al [2] presented a systematic process to supply position in multi-mode MIMO antennas based on the characteristic mode theory. For multi-mode MIMO antennas, a circuit model exploiting modal radiated power was developed. Additionally, optimal feed positions were decided to stimulate these modes exploiting the reflection coefficient of the antenna and modal radiated power. In 2019, Parvez Shaik et al [3], worked on a dual-hop cooperative MIMO system performance for several futuristic QAM methods. A non-regenerative MIMO scheme with the TAS method was considered whereas a single transmit antenna. Taking into consideration of system model, it was presumed that relay, source, and a destination, were equipped by means of multiple antennas. In 2020, SalmanKhalid et al [4], a joint TAS and a precoding model was proposed. To carry out antenna selection to restrict the count of active antennas low Complexity heuristic techniques were exploited. Therefore, while minimizing the power utilization the energy efficiency will increase. In 2020, Omid Yazdani et al [5], proposed a novel and competent TAS for D2D mmWave networks on the basis of the Lagrangian relaxation technique. To examine the performance of the developed method another competent low-complexity greedy-based ASRA method was developed.

\section{System Model}

Let a single isolated cell encompass $\mathrm{B}$ terminals and a single BS. In $\mathrm{E}_{\mathrm{t}}$ numbers, the transmitting (TX) antennas are incorporated in Base Station. Each B contains an individual RX antenna.

At terminals Eq. (1) indicates received signal vectors, here for each $B$ the sindicates $B \times 1$ received vector, For forwarding link $\mathrm{P}_{\mathrm{tx}}$ indicates complete TX power, $\mathrm{O}$ denotes $\mathrm{B} \times \mathrm{E}_{\mathrm{t}}$ small scale Rayleigh fading channel matrix among $\mathrm{E}_{\mathrm{t}} \mathrm{BS}$ antennas and $B$ terminals, $\varsigma$ indicates TX power normalization parameter, which is calculated using $\varsigma \approx \sqrt{\frac{E_{t}}{B}} . V$ indicates $E_{t} \times B$ precoding matrix to reduce IUI, sv indicates the $B \times 1$ message signal vector, and nvindicates $B \times 1 \quad A W G N$ noise vector. By using precoding matrices, for LS-MIMO, two common pre-coding matrices are used, $\left(\mathrm{ZF}: \mathrm{V}=\mathrm{A}^{\mathrm{A}}\left(\mathrm{AA}^{\mathrm{A}}\right)^{-1}\right) \mathrm{ZF}$ and $M F\left(M F: V=E_{t}^{-1} A^{A}\right)$, are devised. OFDM is used as the narrowband signal and it might effectively change the wideband signal to a narrowband signal. 


$$
\mathrm{s}_{\mathrm{m}}=\sqrt{\mathrm{P}_{\mathrm{tx}}} \mathrm{A} \varsigma \mathrm{Vsv}+\mathrm{nv}
$$

Consider transmitter possess idyllist CSI. The system is indicated as LS-MIMO while $\mathrm{E}_{\mathrm{t}}>10 \mathrm{~B}$ is based on norms of thumb.

To calculate LS-MIMO EE organized green BS, there is a requirement of deciding an appropriate power consumption model. Eq. (2) indicates summation power, $\mathrm{P}_{\text {sum }}$, a tractable power utilization technique integrating exact significant LSMIMO power consumption elements, whereas baseband power consumption is denoted as $\mathrm{P}_{\mathrm{BA}}$, $\mathrm{PA}$ power consumption is denoted as $\mathrm{P}_{\mathrm{PA}}$, and $\mathrm{RF}$ front-end power utilization which is encompassed of a mixer, filter, DAC power utilization is denoted as $\mathrm{P}_{\mathrm{RF}_{\text {front }}}$.

$$
\mathrm{P}_{\text {sum }}=\mathrm{P}_{\mathrm{PA}}+\mathrm{P}_{\mathrm{BA}}+\mathrm{E}_{\mathrm{t}} \mathrm{P}_{\mathrm{RF}} \text { front }
$$

For analytic ease, computation of power consumption $\mathrm{P}_{\mathrm{cp}}$ is done which increases with the maximize in $\mathrm{E}_{\mathrm{t}}$ as denotes exploiting Eq. (3). Hence, Eq. (3) is denoted as Eq. (4).

$$
\begin{aligned}
& \mathrm{P}_{\mathrm{cp}}=\left(\mathrm{P}_{\mathrm{BA}} / \mathrm{E}_{\mathrm{t}}+\mathrm{P}_{\mathrm{RF}_{\text {front }}}\right) \\
& \mathrm{P}_{\text {sum }}=\mathrm{P}_{\mathrm{PA}}+\mathrm{E}_{\mathrm{t}} \mathrm{P}_{\mathrm{cp}}
\end{aligned}
$$

Furthermore presumes OFDM system (10MHz) encompassing subcarriers (1024) by means of higher Class-B effectual (78:5\%), and IBO as $11 \mathrm{~dB}$. Based on these constraints, the effectiveness of PA is chosen as $22 \%$. Based on the outsized antennas number, the PA equation with increased effectuality might be costly. The relationship among $\mathrm{P}_{\mathrm{PA}}$ and $\mathrm{P}_{\mathrm{tx}}$ is indicated exploiting Eq. (5).

$$
\mathrm{P}_{\mathrm{tx}}=\eta \mathrm{P}_{\mathrm{PA}}
$$

LS-MIMO baseband power utilization model because of $\mathrm{P}_{\mathrm{BA}} \quad \mathrm{sv}(\mathrm{R}$ flops $) \mathrm{P}_{\mathrm{BA}}$ is indicated as explained in [6] [7].

$$
\mathrm{Z}=\mathrm{E}_{\mathrm{t}} \mathrm{C} \cdot\left[\begin{array}{l}
\left(\frac{\mathrm{S}_{\mathrm{gi}}}{\mathrm{S}_{\mathrm{sd}}}\right) \log _{2}\left(\mathrm{~S}_{\mathrm{gi}} \mathrm{C}\right)+\left(\frac{\mathrm{S}_{\mathrm{gi}}}{\mathrm{S}_{\mathrm{sd}}}\right)\left(1-\frac{\mathrm{S}_{\mathrm{pt}}}{\mathrm{S}_{\mathrm{st}}}\right) \mathrm{B}+\left(\frac{\mathrm{S}_{\mathrm{gi}}}{\mathrm{S}_{\mathrm{sd}}}\right) \\
\left(\frac{\mathrm{S}_{\mathrm{pt}}}{\mathrm{S}_{\mathrm{st}}}\right) \log _{2}\left(\frac{\mathrm{S}_{\mathrm{gi}} \mathrm{S}_{\mathrm{pt}}}{\mathrm{S}_{\mathrm{sd}} \mathrm{S}_{\mathrm{dl}}}\right)+\left(\frac{\mathrm{S}_{\mathrm{dl}}}{\mathrm{S}_{\mathrm{st}}}\right) \mathrm{B}^{2}
\end{array}\right]
$$

Within Eq. (6), each constraint is described as below

- The parameter $\mathrm{C}$ indicates the bandwidth possessing $10 \mathrm{MHz}$ power utilization.

- The parameter $\mathrm{S}_{\text {st }}$ denotes the slot length possessing $0.5 \mathrm{~ms}$ power utilization.

- The parameter $\mathrm{S}_{\mathrm{pt}}$ indicates the pilot length within 1 slot possessing $0.214 \mathrm{~ms}$ power utilization.

- The parameter $\mathrm{S}_{\mathrm{sd}}$ denotes the symbol duration possessing $0.214 \mathrm{~ms}$ power utilization.

- The parameter $\mathrm{S}_{\mathrm{co}}$ denotes GI which posses $4.7 \mu$ s power utilization.

- The parameter $\mathrm{S}_{\mathrm{gi}}$ denotes the Symbol without GI possessing $66.7 \mu$ s power utilization.

- The parameter $\mathrm{S}_{\mathrm{dl}}$ indicates the delay spread GI possessing $4.7 \mu \mathrm{s}$ power utilization.

In [8] these constraints were explained and the LTE system in [9]. The correlation among $P_{B A}$ and sv(Rflops)is denoted in Eq. (7).

$$
\mathrm{P}_{\mathrm{BA}}=\frac{\mathrm{sv}(\mathrm{R} \text { flops })}{\varpi(\mathrm{R} \text { flops } / \mathrm{U})}
$$

Here, the efficacy of VLSI processing is denoted by $\varpi$, and $\varpi=5 R$ flop/U and 50Rflop/Uwas chosen

\subsection{Optimal TAS Selcetion}

As per eq. (8), the symbol used by $\mathrm{m}^{\text {th }}$ user is indicated, in that the $1 \times \mathrm{E}_{\mathrm{t}}$ channel vector is indicated as $\mathrm{g}_{\mathrm{k}}$ : for $\mathrm{m}^{\text {th }}$ user and $\mathrm{E}_{\mathrm{t}} \times 1$ precoding vector is described using $\mathrm{p}:_{\mathrm{k}}$ for $\mathrm{m}^{\text {th }}$ user. The last variable attained by Eq. (8) is called IUI.

$$
\mathrm{q}_{\mathrm{k}}=\sqrt{\frac{\mathrm{P}_{\mathrm{tx}} \mathrm{E}_{\mathrm{t}}}{\mathrm{B}}} \mathrm{g}_{\mathrm{k}}, \mathrm{p}: \mathrm{r}_{\mathrm{k}} \mathrm{z}_{\mathrm{k}}+\mathrm{e}_{\mathrm{k}}+\sqrt{\frac{\mathrm{P}_{\mathrm{tx}} \mathrm{E}_{\mathrm{t}}}{\mathrm{B}}} \sum_{\mathrm{l} \neq \mathrm{k}} \mathrm{g}_{\mathrm{k}},: \mathrm{p}:,_{1} \mathrm{z}_{1}
$$

As per eq. (9), the single isolated cell size is calculated, in that the scaling factor for pilot overhead and GI is indicated as $\alpha$ and noise power in a particular bandwidth $\mathrm{C}$ is indicated as $\mathrm{E}_{0} \mathrm{C}$. 
Billiards Inspired Optimization Algorithm for Optimal Antenna Selection

$$
\mathrm{F}=\alpha \mathrm{C} \cdot \sum_{\mathrm{k}=1}^{\mathrm{K}} \mathrm{D}\left[\log _{2}\left(1+\frac{\frac{\mathrm{P}_{\mathrm{tx}} \mathrm{E}_{\mathrm{t}}}{\mathrm{B}}\left|\mathrm{g}_{\mathrm{k}},: \mathrm{p}:,_{\mathrm{k}}\right|^{2}}{\left.\frac{\mathrm{P}_{\mathrm{tx}} \mathrm{E}_{\mathrm{t}}\left|\sum_{\mathrm{l} \neq \mathrm{k}} \mathrm{g}_{\mathrm{k}},: \mathrm{p}:,_{1}\right|^{2}+\mathrm{E}_{0} \mathrm{C}}{\mathrm{B}}\right)}\right]\right.
$$

Eq. (9) is rewritten as Eq. (10) when the system enters at LS MIMO area, (i.e $\mathrm{E}_{\mathrm{t}}>10 \mathrm{~B}$ ), whereas L indicates IUI. The removal of IUI is carried out simply in ZF precoding. The term EE is performed exploiting Eq. (11), which is seen as the most important objective model of experimented technique which requires to be maximized.

$$
\begin{aligned}
& \mathrm{C}_{\text {approx }}^{\mathrm{LS}-\text { MIMO }} \approx \alpha \mathrm{CB} .\left[\log _{2}\left(1+\frac{\mathrm{P}_{\mathrm{tx}} \mathrm{E}_{\mathrm{t}}}{\left(\mathrm{L}+\mathrm{E}_{0} \mathrm{C}\right) \mathrm{B}}\right)\right] \\
& \mathrm{EE}=\mathrm{F} / \mathrm{P}_{\text {sum }}
\end{aligned}
$$

Let $\mathrm{M}_{\mathrm{t}}^{\mathrm{opt}}$ represents the optimal number of TX antenna and which requires to satisfy eq. (12).

$$
\begin{aligned}
& \frac{\partial}{\partial \mathrm{E}_{\mathrm{t}}} \mathrm{EE}=\frac{\partial}{\partial \mathrm{E}_{\mathrm{t}}}\left(\frac{\mathrm{F}_{\text {approx }}}{\frac{1}{\eta} \mathrm{P}_{\mathrm{tx}}+\mathrm{E}_{\mathrm{t}} \mathrm{P}_{\mathrm{cp}}}\right) \\
& =\frac{\mathrm{CBP}_{\mathrm{tx}}}{\left(\mathrm{L}+\mathrm{E}_{0} \mathrm{C}\right) \mathrm{B}\left(1+\frac{\mathrm{P}_{\mathrm{tx}} \mathrm{E}_{\mathrm{t}}}{\left(\mathrm{L}+\mathrm{E}_{0} \mathrm{C}\right) \mathrm{B}}\right) \mathrm{P}_{\text {sum }} \log _{2} \mathrm{p}} \\
& \frac{\mathrm{CBP}_{\mathrm{cp}} \cdot \log _{\mathrm{e}}\left(1+\frac{\mathrm{P}_{\mathrm{tx}} \mathrm{E}_{\mathrm{t}}}{\left(\mathrm{L}+\mathrm{E}_{0} \mathrm{C}\right) \mathrm{B}}\right)}{\mathrm{P}_{\text {sum }}^{2} \log _{2} \mathrm{p}} \\
& =0
\end{aligned}
$$

Hence, the calculation $\mathrm{E}_{\mathrm{t}}^{\mathrm{opt}}$ is performed in Eq. (13), in that $\Gamma=\frac{\mathrm{P}_{\mathrm{tx}}^{2}-\mathrm{BE}_{0} \mathrm{CP}_{\mathrm{cp}}-\mathrm{BLP}_{\mathrm{cp}}}{\mathrm{B}\left(\mathrm{L}+\mathrm{E}_{0} \mathrm{C}\right) \mathrm{P}_{\mathrm{cp}} \exp (1)}$ and U indicates lambert $\mathrm{U}$ function which is stated as $\Gamma=\mathrm{U}(\Gamma) \exp (\mathrm{U}(\Gamma))$.

$$
\mathrm{E}_{\mathrm{t}}^{\mathrm{opt}} \approx \frac{\left(\mathrm{L}+\mathrm{E}_{0} \mathrm{C}\right) \mathrm{B}}{\mathrm{P}_{\mathrm{tx}}}(-1+\exp (1+\mathrm{U}(\Gamma)))
$$

In Eq. (13), $\mathrm{E}_{\mathrm{t}}^{\text {opt }}$ can be obtained easily and the important problem in this is regarding chosen of the $\mathrm{E}_{\mathrm{t}}^{\mathrm{opt}}$ antenna's number from a complete number of antenna, $\mathrm{E}_{\mathrm{t}}^{\mathrm{total}}$. The predefined count of antennas of $\mathrm{E}_{\mathrm{t}}^{\mathrm{total}}$ is chosen using a conventional selection of antenna methods as channel capability maximization mode. When selecting columns of $A$, channel ability of $E_{t}^{\text {opt }}$ chosen antenna system, is $\left\{b_{1}, b_{2}, \ldots . . b_{E_{t}^{\text {opt }}}\right\}$, $\mathrm{F}_{\left\{\mathrm{b}_{1}, \mathrm{~b}_{2}, \ldots . \mathrm{b}_{\mathrm{t}}^{\text {opt }}\right\}}$ is indicated by eq. (14), in that the $1 \times \mathrm{E}_{\mathrm{t}}^{\text {opt }}$ channel vector for $k^{\text {th }}$ user is represented as $\left.\mathrm{g}_{\mathrm{k},\left\{\mathrm{b}_{1}, \mathrm{~b}_{2}, \ldots . \mathrm{b}_{\mathrm{E}_{\mathrm{t}}^{\text {opt }}}\right\}}\right\}, \mathrm{E}_{\mathrm{t}}^{\text {opt }} \times 1$ precoding vector for $\mathrm{k}^{\text {th }}$ user is shown as $\mathrm{p}_{\left\{\mathrm{b}_{1}, \mathrm{~b}_{2}, \ldots . \mathrm{b}_{\left.\mathrm{E}_{\mathrm{t}}^{\text {opt }}\right\}}\right\}, \mathrm{k}}$ while $\left\{\mathrm{b}_{1}, \mathrm{~b}_{2}, \ldots . . \mathrm{b}_{\mathrm{E}_{\mathrm{t}}^{\text {opt }}}\right\}^{\text {th }}$ is chosen.

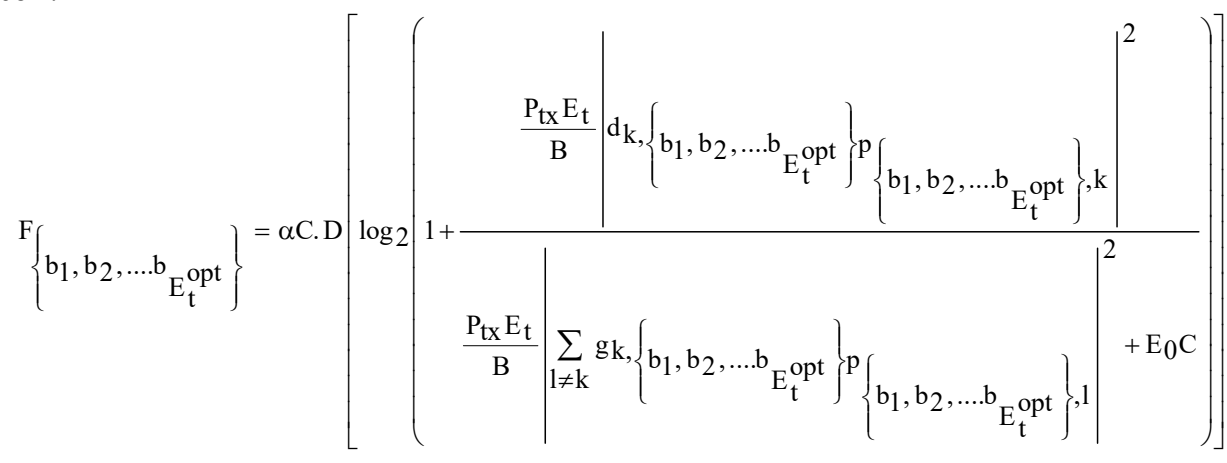


From the green BS perspective, EE requires an increase sooner than increasing ability. In this scenario, from Eq. (11), for EE, optimum AS, and optimum AS for channel ability is similar, as due to the $\mathrm{E}_{\mathrm{t}}^{\text {opt }}$ was selected preceding from (13) for EE consequently the $\mathrm{P}_{\text {sum }}$ presumed to be independent of AS. To attain optimum chosen of the antenna, selection of $\mathrm{E}_{\mathrm{t}}^{\mathrm{opt}}$ columns are to be prepared for higher EE as mentioned in Eq. (15), in that, whole set possible integration of $\mathrm{E}_{\mathrm{t}}^{\text {opt }}$ antennas is stated as $\mathrm{X}$.

$$
\begin{aligned}
& \left\{b_{1}, b_{2}, \ldots . . b_{E_{t}^{\text {opt }}}\right\}
\end{aligned}
$$

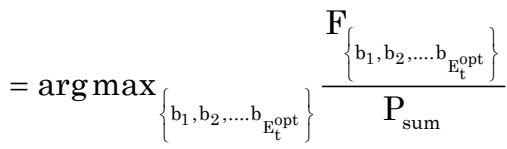

\section{Proposed Billiards Inspired Optimization Algorithm}

The physics behind billiards chiefly engrosses collisions among balls. Collision among two billiards balls is approximately elastic [12]. The ball's kinetic energies are preserved in completely elastic collisions, before and after the collision, and a summation of both momenta. While 2 balls contact each other, forces among balls at some collision point are directed beside an imaginary line which links their centers, like an impact line. With respect to this reality, impact velocities decompose in two modules, a parallel module and a perpendicular module. The initial one is parallel to the balls impact line and next module is perpendicular to it [10]. During a collision, the linking vector initiates from the center of the initial colliding ball to the next ball center [11].

The unit vector of the linking vector is stated as Eq. (16), whereas $r_{1}, r_{2}$ are radii of balls.

$$
\mathrm{e}=\frac{\overrightarrow{\mathrm{m}_{1} \mathrm{~m}_{2}}}{\mathrm{r}_{1}, \mathrm{r}_{2}}
$$

By presuming that this collision is entirely elastic, kinetic energy, and conservation of momentum formulations stated in this system, possesses below form:

$$
\begin{aligned}
& \frac{1}{2} \mathrm{~m}_{1} \mathrm{u}_{1 \mid}^{2}+\frac{1}{2} \mathrm{~m}_{2} \mathrm{u}_{2||}^{2}+\frac{1}{2} \mathrm{~m}_{1} \mathrm{u}_{1 \mid \perp}^{2}+\frac{1}{2} \mathrm{~m}_{2} \mathrm{u}_{2 \mid \perp}^{2} \\
& =\frac{1}{2} \mathrm{~m}_{1} \mathrm{u}_{1||}^{2}+\frac{1}{2} \mathrm{~m}_{2} \mathrm{u}_{2||}^{2}+\frac{1}{2} \mathrm{~m}_{1} \mathrm{u}_{1 \mid \perp}^{2}+\frac{1}{2} \mathrm{~m}_{2} \mathrm{u}_{2 \mid \perp}^{2} \\
& \mathrm{~m}_{1} \mathrm{u}_{1|| \mid}+\mathrm{m}_{2} \mathrm{u}_{2|| \mid}=\mathrm{m}_{1} \mathrm{u}_{1|| \mid}^{\prime}+\mathrm{m}_{2} \mathrm{u}_{2||}^{\prime} \\
& \mathrm{m}_{1} \mathrm{u}_{1 \perp||}+\mathrm{m}_{2} \mathrm{u}_{2 \perp||}=\mathrm{m}_{1} \mathrm{u}_{1 \mid \perp}^{\prime}+\mathrm{m}_{2} \mathrm{u}_{2|| \perp \mid}^{\prime}
\end{aligned}
$$

Whereas, $\mathrm{u}_{1}, \mathrm{u}_{2}$ are first and second ball velocities before the collision and $\mathrm{u}_{1}^{\prime}, \mathrm{u}_{2}^{\prime}$ denotes the velocities of them after the collision. Additionally, symbols || and $\perp$ indicate parallel and perpendicular modules, correspondingly. The parameters $\mathrm{m}_{1} \mathrm{~m}_{2}$ indicate masses of balls.

The perpendicular and parallel modules of the impact velocities are stated as below:

$$
\begin{array}{ll}
\overrightarrow{\mathrm{u}}_{1 \cdot \mid}=\left(\overrightarrow{\mathrm{u}_{1}} \cdot \overrightarrow{\mathrm{e}_{||}}\right) \overrightarrow{\mathrm{e}_{\mid}} & \overrightarrow{\mathrm{u}}_{\bullet_{||}}=\overrightarrow{\mathrm{u}_{1}}-\overrightarrow{\mathrm{u}_{||}} \overrightarrow{\mathrm{e}_{\mid}} \\
\overrightarrow{\mathrm{u}}_{2 \cdot \mid}=\left(\overrightarrow{\mathrm{u}_{2}} \cdot \overrightarrow{\mathrm{e}_{||}}\right) \overrightarrow{\mathrm{e}_{\mid}} & \overrightarrow{\mathrm{u}}_{2 \cdot||}=\overrightarrow{\mathrm{u}_{2}}-\overrightarrow{\mathrm{u}_{||}} \overrightarrow{\mathrm{e}_{\mid}}
\end{array}
$$

As forces are used beside impact line merely, perpendicular modules of momenta are conserved for every ball independently; hence, perpendicular modules of velocities stay unaltered.

$$
\begin{aligned}
& \mathrm{m}_{1} \overrightarrow{\mathrm{u}}_{1 . \perp}=\mathrm{m}_{1} \overrightarrow{\mathrm{u}}_{1 . \perp} \\
& \mathrm{m}_{2} \overrightarrow{\mathrm{u}}_{2 . \perp}=\mathrm{m}_{2} \overrightarrow{\mathrm{u}}_{2 . \perp}^{\prime}
\end{aligned}
$$

Likewise, the elements of kinetic energies in line with perpendicular components stay unaltered before and after collision. Hence, the summation of kinetic energies in line with parallel modules of velocities is preserved too:

$$
\begin{aligned}
& \frac{1}{2} \mathrm{~m}_{1} \mathrm{u}_{1 \mid}^{2}+\frac{1}{2} \mathrm{~m}_{2} \mathrm{u}_{2||}^{2} \\
& =\frac{1}{2} \mathrm{~m}_{1} \mathrm{u}_{1||}^{2}+\frac{1}{2} \mathrm{~m}_{2} \mathrm{u}_{2||}^{2}
\end{aligned}
$$


In the same way, the summation parallel modules of momenta are too conserved.

$$
\mathrm{m}_{1} \mathrm{u}_{1 \mid}+\mathrm{m}_{2} \mathrm{u}_{2||}^{\prime}=\mathrm{m}_{1} \mathrm{u}_{1||}+\mathrm{m}_{2} \mathrm{u}_{2||}^{\prime} \text {. }
$$

Eq. (23) and (24) with a small manipulation must be reformulate as:

$$
\begin{aligned}
& \mathrm{m}_{1}\left(\mathrm{u}_{1 \mid}^{2}-\mathrm{u}_{1 \mid \|}^{2}\right)_{\|}=\mathrm{m}_{2}\left(\mathrm{u}_{2 \mid}^{2}-\mathrm{u}_{2 \|}^{2}\right)_{\|} \\
& \mathrm{m}_{1}\left(\mathrm{u}_{1 \mid}-\mathrm{u}^{\prime} \|\right)_{\|}=\mathrm{m}_{2}\left(\mathrm{u}_{2 \mid}^{\prime}-\mathrm{u}_{2 \| \mid}\right)_{\|}
\end{aligned}
$$

Eq. (25) is reformulated as:

$$
\mathrm{m}_{1}\left(\mathrm{u}_{1 \mid}-\mathrm{u}^{\prime}||\right)\left(\mathrm{u}_{1 \mid}+\mathrm{u}^{\prime}|1 .| \|\right)=\mathrm{m}_{2}\left(\mathrm{u}_{2.1}^{\prime}-\mathrm{u}_{2.1}^{\prime}||\right)\left(\mathrm{u}_{2 \mid}^{\prime}+\mathrm{u}_{2.1}\right)
$$

By dividing Eq. (27) by Eq. (26),

$$
\mathrm{u}_{1.1 \mid}+\mathrm{u}^{\prime}|1 .|||\left|=\mathrm{u}_{2.1 \mid}+\mathrm{u}^{\prime}\right| 2 \cdot|||| \cdot
$$

This exhibits that summation of parallel velocities before and after collision are equivalent. Based on Eq. (24) and (28), concluding parallel modules of velocities subsequent to the collision are indicated as:

$$
\begin{aligned}
& \mathrm{u}^{\prime}|1 .| \| \mid=\frac{\mathrm{m}_{1}-\mathrm{m}_{2}}{\mathrm{~m}_{1}+\mathrm{m}_{2}} \mathrm{u}_{1 . \|}+\frac{2 \mathrm{~m}_{2}}{\mathrm{~m}_{1}+\mathrm{m}_{2}} \mathrm{u}_{1 . \|} \\
& \mathrm{u}^{\prime}|2 .| \| \mid=\frac{2 \mathrm{~m}_{1}}{\mathrm{~m}_{1}+\mathrm{m}_{2}} \mathrm{u}_{1 . \|}+\frac{\mathrm{m}_{2}-\mathrm{m}_{1}}{\mathrm{~m}_{1}+\mathrm{m}_{2}} \mathrm{u}_{2 . \|}
\end{aligned}
$$

If balls possess equivalent masses, eq. (29) and (30) turn out to be as follow, depicting that balls switch merely the parallel module of their velocities, even though perpendicular modules stay unchanged.

$$
\mathrm{u}^{\prime}|1 .||| \mid=\mathrm{u}_{2.1 \mid} \cdot \mathrm{u}_{2 .|| \mid}^{\prime}=\mathrm{u}_{|1 .||| \mid} \cdot
$$

The ultimate velocity vectors are calculated by summation of both modules as

$$
\begin{aligned}
& \overrightarrow{u^{\prime}|1 .|}=u_{1 . \| \mid}^{\prime} \cdot \overrightarrow{e^{\prime}|| \cdot||}+\overrightarrow{u^{\prime}|1 .|} \\
& \overrightarrow{u^{\prime} 2 . \mid}=u_{2 . \|}^{\prime} \cdot \overrightarrow{e^{\prime}|| \cdot \mid}+\overrightarrow{u^{\prime}|2 .|}
\end{aligned}
$$

Aforesaid ideas and details may be widespread to collisions in multi-dimensional spaces. While two balls collide together, the force among the balls is directed beside an imaginary line which links their centers. Hence, besides this direction, there is merely a one-dimensional collision issue, and velocities in this direction are computed by Eq. (29) and (30) and other modules of velocities remain unaltered.

As stated before, the developed meta-heuristic approach is inspired by the billiards game.

Step 1: Initialization: The initial population of balls is distributed arbitrarily in the search space as:

$$
\begin{aligned}
& \mathrm{B}_{\mathrm{n}, \mathrm{m}}^{0}=\operatorname{var}_{\mathrm{m}}^{\min }+\operatorname{rand}_{(0,1)}\left(\operatorname{var}_{\mathrm{m}}^{\max }-\operatorname{var}_{\mathrm{m}}^{\min }\right) . \mathrm{n}=1,2,3 \ldots \\
& \quad 2 \mathrm{~N} ; \mathrm{m}=1,2,3, \ldots \mathrm{M}
\end{aligned}
$$

In eq. (34), $\mathrm{B}_{\mathrm{n}, \mathrm{m}}^{0}$ indicates the initial value of the $\mathrm{m}^{\text {th }}$ variable of $\mathrm{n}^{\text {th }}$ ball. $\operatorname{var}_{\mathrm{m}}^{\max }$ and $\operatorname{var}_{\mathrm{m}}^{\min }$ indicates the utmost and least permissible values for $\mathrm{m}^{\text {th }}$ variable. rand $_{(0,1)}$ indicates an arbitrary number with uniform distribution in the interval $[0,1]$, and $\mathrm{M}$ and $2 \mathrm{~N}$ indicates the numeral of agents and variables, correspondingly. In addition, few pockets are produced by the preceding equation arbitrarily in search space. The user determines the numeral of pockets.

Step 2: Evaluation: On the basis of the objective function, the positions of the balls and pockets' are evaluated.

Step 3: In this algorithm to determine pockets BOA pockets play two roles, an objective for balls that offers the exploitation capability of the method and a memory that stores the first top solutions found hitherto. Continuation of this memory enhances the performance of the technique without raising the evaluation cost. In each iteration, this memory is updated and substituted with optimal balls found locations.

Step 4: Grouping balls: Subsequent to the determination, based on their finesses, the pockets, balls are sorted and subsequently they are divided into two equivalent groups, ordinary balls and cue balls. The higher half comprises ordinary balls (i. e. $\mathrm{n}=1, \ldots \ldots, \mathrm{N}$ ) and second one decides cue balls (i. e. $\mathrm{n}=\mathrm{N}+1, \ldots ., 2 \mathrm{~N})$ 


\section{Result and Discussion}

\subsection{Experimentation Setup}

The experimentation of the applied optimal antenna selection model was carried out exploiting MATLAB, and outcomes were analyzed. Furthermore, the developed method regarding the performance was differentiated over other conventional techniques such as GA [6], ABC [7], PSO [13], and FF [14]. The experimentation was carried out with two setups. The initial setup was $\mathrm{P}_{\mathrm{tx}}=40 \mathrm{~W}$,

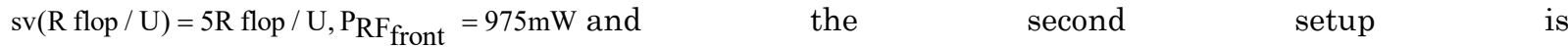
$\mathrm{P}_{\mathrm{tx}}=10 \mathrm{~W}, \mathrm{P}_{\mathrm{sum} 1} \mathrm{sv}(\mathrm{R}$ flop $/ \mathrm{U})=50 \mathrm{R}$ flop $/ \mathrm{U}, \mathrm{P}_{\mathrm{RF}}$ front $=97.5 \mathrm{~mW}$. The experimentation analysis was performed regarding the optimal count of the antenna were performed.

\subsection{Relative Energy Efficiency Analysis}

Table 1 and 2 exhibits the analysis of relative EE for the proposed method over existing methods for setup 1 and 2. Two sets of experiments have experimented with respect to the number of users are experimenting with the conventional methods. The analysis, therefore, summarized that for 1st and 2nd set of experiments, under 40 users, the proposed method is obtained by means of better EE than conventional techniques like $\mathrm{ABC}, \mathrm{GA}, \mathrm{FF}$, and PSO.

Table1. Performance Analysis of Proposed And Existing Techniques for Set Up 1

\begin{tabular}{|c|c|c|c|c|c|}
\hline \multicolumn{6}{|c|}{ Match Filtering } \\
\hline No of User & $\mathrm{ABC}$ & GA & FF & PSO & Proposed \\
\hline 5 & 0.84788 & 0.84715 & 0.84758 & 0.84425 & 0.84757 \\
\hline 10 & 0.81041 & 0.80887 & 0.81028 & 0.81024 & 0.81128 \\
\hline 15 & 0.87481 & 0.87482 & 0.87405 & 0.87481 & 0.87788 \\
\hline 20 & 0.84728 & 0.84772 & 0.84184 & 0.84717 & 0.85207 \\
\hline 25 & 0.85528 & 0.85518 & 0.85578 & 0.85524 & 0.89041 \\
\hline 30 & 0.81114 & 0.81111 & 0.81088 & 0.81102 & 0.81152 \\
\hline 35 & 0.81521 & 0.8157 & 0.81525 & 0.81507 & 0.87317 \\
\hline 40 & 0.81815 & 0.81887 & 0.81878 & 0.8184 & 0.87787 \\
\hline \multicolumn{6}{|c|}{ Zero Forcing } \\
\hline No of User & $\mathrm{ABC}$ & GA & FF & PSO & Proposed \\
\hline 5 & 0.84755 & 0.84284 & 0.84784 & 0.84772 & 0.84447 \\
\hline 10 & 0.81021 & 0.81054 & 0.80872 & 0.81027 & 0.81075 \\
\hline 15 & 0.87425 & 0.87421 & 0.87405 & 0.87427 & 0.87785 \\
\hline 20 & 0.84187 & 0.84718 & 0.84718 & 0.84187 & 0.85187 \\
\hline 25 & 0.85578 & 0.85508 & 0.85545 & 0.85577 & 0.81057 \\
\hline 30 & 0.81104 & 0.81142 & 0.8108 & 0.81101 & 0.81171 \\
\hline 35 & 0.81541 & 0.81547 & 0.81527 & 0.81487 & 0.87011 \\
\hline 40 & 0.81878 & 0.81885 & 0.8184 & 0.81875 & 0.87788 \\
\hline
\end{tabular}

Table 2.Performance Analysis of Proposed and Conventional Models for Set Up 2

\begin{tabular}{cccccc}
\multicolumn{7}{c}{ Table 2.Performance Analysis of Proposed and Conventional Models for Set Up 2} \\
\hline \multicolumn{5}{c}{ Match Filtering } \\
No of User & ABC & GA & FF & PSO & Proposed \\
\hline $\mathbf{5}$ & 0.83363 & 0.83376 & 0.83337 & 0.83337 & 0.88001 \\
$\mathbf{1 0}$ & 0.91007 & 0.90983 & 0.91031 & 0.91031 & 0.91933 \\
$\mathbf{1 5}$ & 0.93361 & 0.93386 & 0.93311 & 0.93311 & 0.93779 \\
$\mathbf{2 0}$ & 0.93797 & 0.93788 & 0.93789 & 0.93789 & 0.96816 \\
$\mathbf{2 5}$ & 0.96667 & 0.9667 & 0.96679 & 0.96609 & 0.97631 \\
$\mathbf{3 0}$ & 0.97099 & 0.97136 & 0.97108 & 0.97108 & 0.97011 \\
$\mathbf{3 5}$ & 0.97639 & 0.97637 & 0.97613 & 0.97613 & 0.97377 \\
$\mathbf{4 0}$ & 0.97887 & 0.97891 & 0.97871 & 0.97871 & 0.97733 \\
& & Zero Forcing & & \\
No of User & $\mathbf{A B C}$ & $\mathbf{G A}$ & FF & PSO & Proposed \\
\hline $\mathbf{5}$ & 0.83173 & 0.83197 & 0.83103 & 0.83103 & 0.88068 \\
$\mathbf{1 0}$ & 0.91017 & 0.91066 & 0.90977 & 0.90977 & 0.91837 \\
$\mathbf{1 5}$ & 0.93311 & 0.93318 & 0.9333 & 0.9333 & 0.93731 \\
$\mathbf{2 0}$ & 0.93711 & 0.93717 & 0.93797 & 0.93797 & 0.96771 \\
$\mathbf{2 5}$ & 0.96677 & 0.96633 & 0.96613 & 0.96613 & 0.97396 \\
$\mathbf{3 0}$ & 0.97087 & 0.97099 & 0.97097 & 0.97097 & 0.9799 \\
$\mathbf{3 5}$ & 0.97617 & 0.97639 & 0.97607 & 0.97607 & 0.97371 \\
$\mathbf{4 0}$ & 0.97877 & 0.97881 & 0.97838 & 0.97838 & 0.9773 \\
\hline
\end{tabular}




\section{Conclusion}

The foremost purpose of this article was to propose a new algorithm called billiards inspiration optimization algorithm to choose the optimal TAS via a multi-objective issue that raises both relative EE and capacity. In actual fact, the developed technique was optimally chosen the transmit antennas number and recognizes which antenna to be chosen. At last, the developed method regarding the performance was differentiated and examined over other conventional techniques with respect to some analysis such as relative efficiency, capacity, and optimal antenna selection.

\section{Compliance with Ethical Standards}

Conflicts of interest: Authors declared that they have no conflict of interest.

Human participants: The conducted research follows the ethical standards and the authors ensured that they have not conducted any studies with human participants or animals.

\section{References}

[1] Mahdi EskandariAli Mohamad Doost HoseiniMohammad Sadegh Fazel,"An energy-efficient joint antenna selection and power allocation for MIMO systems under limited feedback",Signal Processing9 May 2019.

[2] Mostafa parvinJavad Ahmadi-Shokouh Hamideh Dashti,"Systematic feed locating in multi-mode MIMO antennas using characteristic mode theory", AEU - International Journal of Electronics and Communications13 August 2020.

[3] Parvez ShaikPraveen K. SingyaVimal Bhatia,"Performance analysis of QAM schemes for non-regenerative cooperative MIMO network with transmit antenna selection",AEU - International Journal of Electronics and Communications20 May 2019.

[4] Salman KhalidRashid MehmoodMuhammad Naeem,"Joint transmit antenna selection and precoding for millimeter wave massive MIMO systems Physical Communication28 May 2020.

[5] Omid YazdaniGhasem MirjalilyMehdi Monemi,"Efficient transmit antenna selection and resource allocation scheme for mmWave D2D networks AEU - International Journal of Electronics and Communications9 June 2020.

[6] Bi-bo HU, Yuan-an LIU, Gang XIE, Jin-chun GAO, Ya-lin YANG," Energy efficiency of massive MIMO wireless communication systems with antenna selection", The Journal of China Universities of Posts and Telecommunications, vol.21, no.6, pp.1-8, December 2014

[7] Masadeh, Raja \& Mahafzah, Basel \& Sharieh, Ahmad," Sea Lion Optimization Algorithm", International Journal of Advanced Computer Science and Applications, volume. 10, page no.388-395, 2019.

[8] Theodoros D. Vrionis, Xanthi I. Koutiva, and Nicholas. A. Vovos,"A Genetic Algorithm-Based Low Voltage RideThrough Control Strategy for Grid Connected Doubly Fed Induction Wind Generators",IEEE Transactions on Power Systems, volume. 29, number.3, May2014.

[9] Kai Zhou Gao, Ponnuthurai Nagaratnam Suganthan, Quan Ke Pan, Mehmet Fatih Tasgetiren, Ali Sadollah, "Artificial bee colony algorithm for scheduling and rescheduling fuzzy flexible job shop problem with new job insertion", Knowledge-Based Systems, volume. 109, page no. 1-16, 1 October 2016.

[10] Landry J-F, Dussault J-P. AI optimization of a billiard player. J Intell Robot Syst 2007;50:399-417.

[11] Teng C. A Mathematical Analysis of Billiards Games : Amplification Factor, Stability of Ball Path, and Player's Pattern. Citeseer 2008.

[12] Čepič M. Elastic collisions of smooth spherical objects: finding final velocities in four simple steps. Am J Phys 2019;87:200-7.

[13] Junhao Zhang, Pinqi Xia, "An improved PSO algorithm for parameter identification of nonlinear dynamic hysteretic models", Journal of Sound and Vibration, volume. 389, page no. 153-167, 17 February 2017.

[14] Hui Wang, Wenjun Wang, Xinyu Zhou, Hui Sun, Zhihua Cui, “ Firefly algorithm with neighborhood attraction”, Information Sciences, volume. 382-383, page no. 374-387, March 2017.

[15] Amol V Dhumane,"Examining User Experience of eLearning Systems using EKhool Learners",Journal of Networking and Communication Systems (JNACS), Volume 3, Issue 4, October 2020. 